\title{
Perfect for patients of all ages
}

A study found that 18-year-olds who used a computer for more than three hours a day tended to neglect their oral health. ${ }^{1}$

Support your younger patients by helping to put the basics in place early: regular appointments, a healthy diet and a good, daily cleaning routine.

TANDEX oral hygiene products are perfect for patients of all ages, including teens.

The brushes are well made, easy to manipulate, robust, yet gentle and give the mouth a thorough clean. Patients may want to add the FLEXI interdental brush, plus PREVENT GEL and mouthwash, for a mouth that's really healthy.

For more information on Tandex's range of products, visit www.tandex.dk.

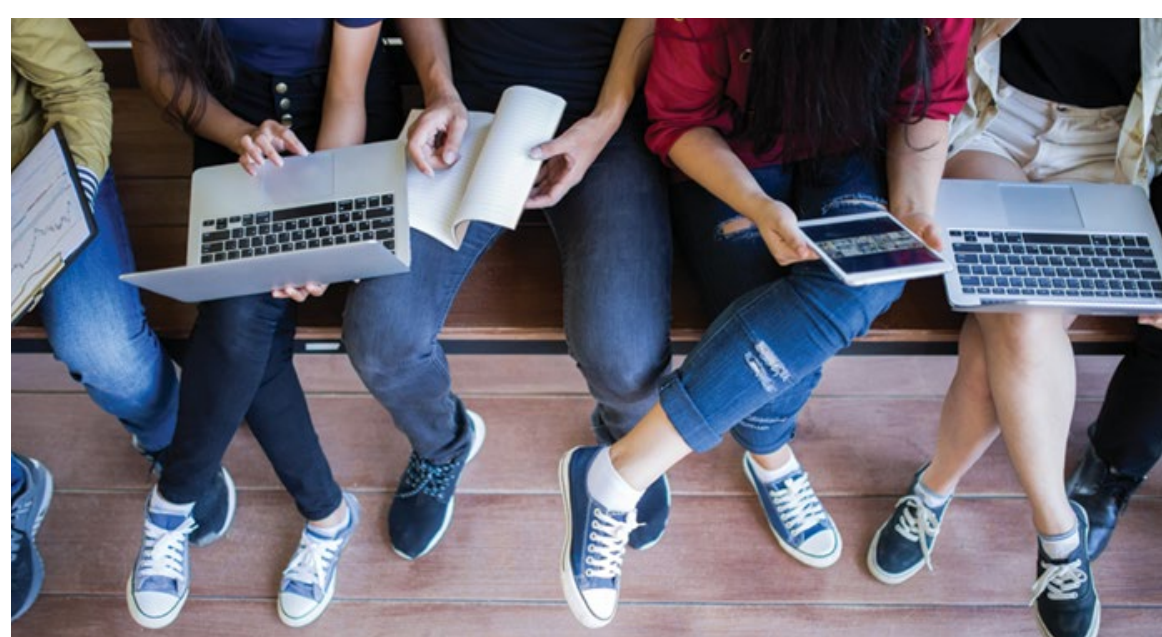

\section{References}

1. Olczak-Kowalczyk D, Tomczyk J, Gozdowski D, Kaczmarek U. Excessive computer use as an oral health risk behaviour in 18-year-old youths from Poland: A cross-sectional study. Clin Exp Dent Res 2019; 5: 284-293.

\section{Improve life after tooth loss}

A removable partial denture (RPD) created with Ultaire AKP quickly restores function, aesthetics and stability with comfort, strength and durability.

With the potential to reduce the impact of tooth loss and preserve quality of life, Ultaire AKP offers your patients a metal-free alternative that is lightweight, biocompatible and completely taste free!

Ultaire AKP is a high-performance polymer that fits perfectly into the digital workflow to produce highly accurate RPDs with a superior fit.

To enjoy increased patient satisfaction, book a Solvay Dental 360 Professional Lunch and Learn at your practice now.

To book a Solvay Dental 360 Professional Lunch and Learn or to find more information Ultaire AKP and Dentivera milling discs, visit www.solvaydental360.com.

\section{The right choice}

With so many electric toothbrushes available on the market, it can be difficult to recommend the right solution to your patients. Oral healthcare specialist Curaprox aims to make the choice simple with the Hydrosonic Pro (previously known as the Hydrosonic Ortho) toothbrush.

It is complete with three compact brush heads featuring ultra-fine CUREN filaments that facilitate fast, easy and thorough cleaning of the teeth and gums. This innovative device also boasts CURACURVE ergonomics that enable the user to manoeuvre the toothbrush into the most ideal position for effective removal of plaque - even in hard-to-reach areas.

With a powerful battery that ensures two weeks of cleaning for four minutes each day, the Hydrosonic Pro toothbrush offers outstanding mechanical and hydrodynamic performance.

For more information call 01480 862084, email info@curaprox.co.uk or visit www. curaprox.co.uk.

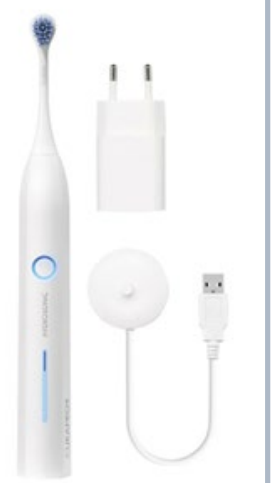

BRITISH DENTAL JOURNAL | VOLUME 227 NO. 8 | OCTOBER 252019

\section{West Yorkshire dental firm} \section{starts world tour}

EthOss Regeneration has kick-started a global tour.

The Silsden-based firm will be showcasing its flagship product, EthOss, at a series of international talks and exhibitions across three continents.

The team will be visiting London, Nigeria, Portugal, Lithuania, the Czech Republic, Morocco, Russia, Estonia, the Netherlands, Greece, Malta, Hungary, Romania, the Philippines, Thailand, Hong Kong and Dubai.

EthOss is made from a mixture of beta tricalcium phosphate and calcium sulphate. The calcium sulphate acts as a built-in barrier against soft tissue ingress, removing the need for an additional collagen membrane - a very popular feature with dentists who often find collagen membranes difficult to handle and an expensive addition to a procedure.

The product is mixed into a paste and applied to the surgical site using a simple syringe delivery system. Once applied, EthOss becomes firm and forms a semi rigid graft providing a highly stable scaffold, leading to impressive clinical success rates of $50 \%$ new bone after 12 weeks.

For more information call $+44(0) 1535$ 843106 or email info@ethoss.dental. 